associated with bilateral temporal polyspike and sharp-wave EEG activity were controlled with phenobarbital. His head circumference was at the 75th percentile and disproportionately large. A repeat ultrasound at 7 mos showed increased echogenicity in the midline with normal ventricles, interpreted as blood in the third ventricle with calcification. CT showed a large midline area of decreased density extending into the lateral ventricles, consistent with a lipoma of the corpus callosum and calcifications. At 18 mos the head is large and development is delayed at the $12 \mathrm{mo}$ level. (Imaizumi so et al. Lesion mistaken for hemorrhage in a premature infant: Lipoma of corpus callosum. Pediatr Neurol Oct 1988; 4:313-6).

COMMENT. Blood, fat, and calcium have similar echogenicity by cranial ultrasound, and all three media are hyperechoic when compared to the moderate echogenicity of cerebral white matter, low echogenicity of gray matter, and absent echogenicity of fluid-filled ventricles or cysts. Lipomas are characterized by hyperechoic densities on cranial sonograms.

\title{
SPINAL CORD HEMANGIOBLASTOMA
}

A 6-month-old infant with a spinal cord hemangioblastoma located in the conus medullaris is reported from the University of Washington School of Medicine, Children's Hospital, Seattle, WA. At birth the physical examination was normal except for a lumbosacral dimple. By 1 mo the dimple had deepened and a hemangioma developed at the site. CT and CT metrizamide myelography revealed a posterior filling defect at the level of the conus medullaris. At laminectomy, a congenital dermal sinus tract, cutaneous capillary hemangioma and cord hemangioblastoma were resected. There were no neurologic deficits either postoperatively or following removal of the tumor. (Michaud LJ et al. Hemangioblastoma of the conus medullaris associated with cutaneous hemangioma. Pediatr Neurol Oct 1988; $\underline{4}: 309-12$ ).

COMMENT. Spinal cord hemangioblastoma rarely presents in infancy and is usually manifest from the third to the fifth decades. It may be associated with a variety of cutaneous and other lesions, including von Hippel-Lindau and Cobb syndromes. Von Hippel-Lindau disease is an autosomal dominant condition characterized by hemangioblastomas of the cerebellum, medulla and spinal cord; angiomas of the retina, liver, and kidney; pheochromocytomas, adenomas, or cysts of the kidney and epididymis; and pancreatic cysts. In Cobb syndrome, cutaneomeningospinal angiomatosis, spinal cord arteriovenous malformations are associated with cutaneous vascular lesions in corresponding dermatomes. These syndromes were not found in the above case-report.

\section{CHEMOTHERAPY FOR MEDULLOBLASTOMA}

The efficacy of adjuvant chemotherapy for patients with 